\title{
Aplicando o Design Science Research no Desenvolvimento de um Sistema Colaborativo Assistivo
}

\section{Daniel Maniglia Amancio da Silva ${ }^{1}$, Deivid Felipe Sartori ${ }^{1}$, Celso Massaki Hirata ${ }^{2}$, Carla Diacui M. Berkenbrock ${ }^{1}$}

\author{
${ }^{1}$ Universidade do Estado de Santa Catarina (UDESC) \\ Joinville - SC - Brasil \\ ${ }^{2}$ Instituto Tecnológico de Aeronáutica (ITA) \\ São José dos Campos - SP - Brasil \\ danielmanilha@hotmail.com, deividfelipi@gmail.com, \\ hirata@ita.br, carla.berkenbrockeudesc.br
}

\begin{abstract}
Resumo. Os avanços da tecnologia, bem como a crescente preocupação da sociedade com a inclusão social de pessoas com deficiências motivaram o desenvolvimento de ferramentas para apoiar pessoas com atrasos cognitivos. Este trabalho tem o objetivo de definir artefatos de comunicação, em smartphones, para um sistema colaborativo de monitoramento geográfico, possibilitando a comunicação do deficiente intelectual com seus cuidadores em seu deslocamentos cotidianos. O trabalho foi guiado pela metodologia de pesquisa Design Science Research (DSR). A pesquisa foi dividida em três ciclos de descoberta de conhecimento: ciclo de conhecimento do usuário, ciclo de design dos artefatos e ciclo de avaliação da usabilidade. O presente artigo também detalha o levantamento de requisitos, desenvolvimento dos artefatos e avaliação do sistema. Como resultados, destaca-se a geração do conhecimento científico proporcionado pela utilização da metodologia de pesquisa DSR. Além disso, o trabalho visa fortalecer a utilização da abordagem de desenvolvimento centrado no usuário e do design participativo no contexto de desenvolvimento de um sistema colaborativo assistivo.
\end{abstract}

\begin{abstract}
Advances in technology and the growing concern of society in relation to the social inclusion of people with disabilities motivated the development of tools to support people with cognitive delays. This work aims to define communication artifacts, used in smartphones, for a collaborative system of geographical monitoring, enabling the communication of intellectual disabled with their caregivers in their daily movements. The work was guided by Design Science Research (DSR). The research was divided into three cycles of knowledge discovery: user knowledge cycle, design cycle of artifacts and usability evaluation cycle. This article also details the requirements gathering, development of artifacts and system evaluation. As a result, there is the generation of scientific knowledge provided by the use of the DSR research methodology. In addition, this work intend to strengthen the use of user centered design and participatory design in the development of a collaborative assistive system.
\end{abstract}




\section{Introdução}

No Brasil, nas últimas décadas, novas políticas públicas têm sido estabelecidas para incentivar o desenvolvimento e uso de Tecnologias Assistivas (TA). Dentre as deficiências legalmente assistidas, pode-se citar a deficiência intelectual.

Deficiência intelectual (DI) é um termo que se usa quando uma pessoa apresenta certas limitações no seu funcionamento mental e no desempenho de tarefas como as de comunicação, cuidado pessoal e de relacionamento social [Almeida 2005].

A vida em comunidade das pessoas com DI tem sido estudada [Fink 1992], [Satcher 2000] , [Wahl 1999] e é evidente o aumento das ligações comunitárias, do suporte social e das oportunidades de recuperação pela socialização.

Sistemas computacionais em rede disseminaram-se por todo o sistema social e vem provocando transformações em todos os setores a vida contemporânea [Fuks 2011]. Portanto, sistemas assistivos e colaborativos podem servir como ferramenta de apoio a colaboração e socialização de pessoas com DI.

Porém, existem desafios no desenvolvimento de tecnologias assistivas e sistemas colaborativos. A característica de multidisciplinaridade, as particularidades, limitações e diferentes necessidades dos usuários tornam complexo o desenvolvimento destes sistemas com foco em "usuários especiais". Para [Druin 2009], desenvolver sistemas é sempre uma tarefa desafiadora e o desafio é ainda maior no desenvolvimento de sistemas para pessoas com necessidades especiais. A combinação de relações de poder, a reduzida habilidade de comunicação e as partes interessadas adicionais, tais como pais, professores ou responsáveis, resulta em uma situação bastante complexa. Já para [Grudin 1994], no desenvolvimento de sistemas, considerar usuários com DI como usuários "vulneráveis" somente evidencia o desconhecimento de métodos adequados de design, a dificuldade na comunicação ou a dificuldade de entender a realidade destes usuários.

A comunicação é um dos pilares dos sistemas colaborativos, uma necessidade para relacionamento social e também uma dificuldade para pessoas com DI. Nesse sentido, o presente artigo detalha a pesquisa em desenvolvimento que busca a geração de conhecimentos sobre a comunicação de pessoas com DI e seus cuidadores. Neste contexto, a Comunicação Aumentativa e Alternativa (CAA) surge como a possibilidade de colaboração envolvendo pessoas com DI. A pesquisa é guiada pela metodologia de pesquisa denominada Desing Science Research (DSR). O presente artigo detalha os ciclos de pesquisa que possibilitaram a construção dos componentes computacionais para possibilitar a comunicação entre pessoas com DI e seus cuidadores em um sistema de monitoramento geográfico.

O restante do artigo está organizado da seguinte forma: a seção 2 apresenta os trabalhos relacionados, a seção 3 apresenta os conceitos de Design Science Recearch e descreve a adaptação realizada para a pesquisa descrita neste artigo. Na seção 4 é feita uma discussão sobre a relevância do problema. As seções 5, 6 e 7 descrevem os artefatos de software gerados na pesquisa, a descrição do desenvolvimento da pesquisa e os ciclos de descoberta de conhecimento e a definição do rigor da pesquisa, respectivamente. Por fim, na seção 8 são apresentadas as considerações finais e os trabalhos futuros. 


\section{Trabalhos Correlatos}

Esta seção apresenta alguns trabalhos diretamente relacionados com a pesquisa descrita no presente artigo. São trabalhos que abordam a utilização do DSR em sistemas colaborativos, avaliação de sistemas para pessoas com deficiências e redes sociais em dispositivos móveis.

[Rocha et al. 2015] utilizaram a metodologia Design Science Research para definição de artefatos para sistemas colaborativos. A pesquisa demonstra a influencia do tamanho do grupo na participação em bate-papo educacional e produz um modelo matemático para estimar a quantidade máxima de alunos que devem participar do bate papo para manter o nível de participação desejado.

[Pries-Heje et al. 2008] apresentam uma discussão sobre a importância da avaliação. No entanto, a discussão de atividades e métodos de avaliação é limitada e, normalmente, assume uma perspectiva posterior, em que a avaliação ocorre após a construção de um artefato. Tais perspectivas podem assumir que a avaliação é um processo empírico e seus métodos podem ser selecionados da mesma forma como métodos de pesquisa empírica. Os autores ainda, demonstram uma gama mais ampla de estratégias de avaliação, que inclui uma avaliação anterior a construção do artefato. Esta visão adiciona ênfase às estratégias para a avaliação de processos de design, além de projetar produtos, utilizando critérios de qualidade.

[Petrie et al. 2006] apresentam dois estudos de casos que exploram técnicas de avaliação assíncrona em ambientes remotos com participantes com deficiência. No trabalho, são utilizadas as técnicas de questionários e observações. O estudo mostra que os dados quantitativos recolhidos em avaliações remotas assíncronas com usuários com deficiência são comparáveis com os dados coletadas nas avaliações locais.

[Chang et al. 2009] sugerem uma arquitetura geral para redes sociais móveis onde os indivíduos nas redes formam uma comunidade virtual, que convergem e se conectam com o outro, com base na comunicação móvel. Os autores também descrevem uma implementação de redes sociais móveis em colaboração com um programa de emprego para as pessoas com doença mental grave.

[de Araújo et al. 2014] utilizaram o design participativo como ferramenta de apoio para o desenvolvimento de um sistema colaborativo para tratamento multidisciplinar na rede do Sistema Único de Saúde. Eles utilizaram técnicas que possibilitaram que os profissionais da saúde, que irão utilizar o sistema, pudessem opinar durante o processo de construção do mesmo.

Assim como o presente trabalho, [Rocha et al. 2015] e [Pries-Heje et al. 2008] utilizam o DSR como guia para descoberta de conhecimento científico no desenvolvimento de sistemas colaborativos. Sendo que para [Rocha et al. 2015] o artefato resultante é uma fórmula matemática para obter o número ideal de participantes em chats e [Pries-Heje et al. 2008] empregam como artefato a avaliação. [de Araújo et al. 2014] utilizam o design participativo para levantamento de requisitos e definição de um sistema colaborativo para a área de saúde. Em [Petrie et al. 2006] é relatada a avaliação, mas no contexto de sistemas onde os usuários são pessoas com deficiências. O contexto do trabalho apresentado por [Chang et al. 2009] tem relação direta com o trabalho relatado neste artigo, pois a pesquisa desenvolvida foca na utilização de redes sociais em dispositivos 
móveis.

A multidisciplinaridade da pesquisa descrita neste artigo justifica a correlação com trabalhos desenvolvidos em contextos diferentes. Nesta seção destacamos trabalhos que utilizam a metodologia DSR para sistemas colaborativos para design e avaliação, assim como trabalhos com deficientes e redes sociais móveis. A junção de partes dos escopos dos trabalhos correlatos adicionado ao foco na Comunicação Aumentativa e Alternativa, define o escopo de trabalho da pesquisa descrita no presente artigo.

\section{Design Science Research}

Design Science Research (DSR) é um método de pesquisa centrado na evolução de uma "ciência do projeto" (design science) originário da área de engenharia de produção, e que evidencia seu sentido e sua forma de operacionalização. Para [Lacerda et al. 2013], o DSR tem foco na importância da definição das classes de problemas e dos artefatos gerados no âmbito da pesquisa. Para [Aken 2004] as classes de problemas podem consistir em uma organização para a trajetória e o desenvolvimento do conhecimento em uma design science. Para [Bax 2015], DSR é uma metateoria que auxilia o pesquisador a criar conhecimento teórico durante os processos de concepção de artefatos, justificando como tais processos podem ser significativos para a comunidade científica.

[Hevner 2007] utiliza o DSR em sistemas de informação. De acordo com o autor, os conhecimentos necessários para realizar uma pesquisa em sistemas de informação envolvem os paradigmas da "ciência do comportamento" e "ciência do design". A ciência do comportamento aborda a pesquisa pelo desenvolvimento de teorias que explicam fenômenos relacionados com a necessidade de negócio identificada e a ciência de design aborda a pesquisa pelo desenvolvimento e avaliação de artefatos projetados para atender a necessidade de negócio identificada.

No contexto da presente pesquisa, a ciência do comportamento guiará o entendimento dos limites de comunicação e entendimento do software pelos usuários com deficiência intelectual e a ciência do design guiará o desenvolvimento de interfaces adaptadas a esses usuários. Portanto, na presente pesquisa, o fenômeno comportamental investigado é a utilização da tecnologia de comunicação por usuários com deficiência intelectual e o artefato desenvolvido são os componentes e recursos de tela adaptados às necessidades dos usuários.

[Hevner 2007] também sugere um framework conceitual, onde define-se que uma pesquisa é realizada em um ambiente produzindo uma base de conhecimento. Os produtos da pesquisa são: teorias e artefatos que devem ser justificados e avaliados para produzirem feedback que possa refinar as teorias e os artefatos. Adicionalmente, o autor disponibiliza um conjunto de diretrizes que as pesquisas em sistema de informação devem apresentar. $\mathrm{Na}$ Tabela 1 estão as diretrizes aplicadas a presente pesquisa.

\section{Relevância do Problema}

Pessoas com DI diariamente realizam trajetos, como por exemplo, ida e volta até a padaria, supermercado ou até mesmo para as empresas onde trabalham. Na maioria das vezes, devido a suas deficiências, essas pessoas dependem da supervisão presencial dos cuidadores na realização de seus trajetos. 
Tabela 1. Diretrizes do DSR adaptado para a pesquisa

\begin{tabular}{|c|c|}
\hline Diretriz & Aplicação da Diretriz \\
\hline $\begin{array}{l}\text { Relevância } \\
\text { do } \\
\text { Problema }\end{array}$ & $\begin{array}{l}\text { "Para as pessoas sem deficiência a tecnologia torna as coisas mais fáceis. } \\
\text { Para as pessoas com deficiência, a tecnologia torna as coisas possíveis" } \\
\text { [Radabaugh 1993]. } \\
\text { Segundo [SNPD 2012] 1,4 por cento da população brasileira tem deficiência } \\
\text { mental ou intelectual. } \\
\text { A presente pesquisa tem foco na colaboração, provida por tecnologia, } \\
\text { para o aumento da autonomia de pessoas com deficiência intelectual }\end{array}$ \\
\hline Artefato & $\begin{array}{l}\text { O artefato produzido são as telas e os componentes de telas (de smartphones) } \\
\text { por onde pessoas com deficiência poderão se comunicar com seus cuidadores }\end{array}$ \\
\hline $\begin{array}{l}\text { Processo de } \\
\text { Busca } \\
\text { da Solução }\end{array}$ & $\begin{array}{l}\text { Guiado pelo método de pesquisa Design Science Research utilizou-se } \\
\text { conceitos de IHC, mais precisamente do Design Centrado no Usuário } \\
\text { e do Design Participativo para pesquisa e desenvolvimento da solução }\end{array}$ \\
\hline $\begin{array}{l}\text { Rigor da } \\
\text { Pesquisa }\end{array}$ & $\begin{array}{l}\text { Para cada ciclo de conhecimento da pesquisa um ou mais conceitos } \\
\text { foram utilizados para garantir o rigor da pesquisa. Avaliação dos especialistas, } \\
\text { Workshops, observação do uso e avaliação da utilização dos componentes } \\
\text { foram utilizados para garantir o rigor da pesquisa durante os ciclos de design }\end{array}$ \\
\hline Avaliação & $\begin{array}{l}\text { Avaliação de usabilidade, com o envolvimento dos usuários. Utilizando a } \\
\text { análise de observação de uso e a análise de utilização de componentes }(\log s)\end{array}$ \\
\hline $\begin{array}{c}\text { Contribuições } \\
\text { da } \\
\text { Pesquisa }\end{array}$ & $\begin{array}{l}\text { Fortalecimento do uso do Design Science Research na área de Sistemas } \\
\text { Colaborativos } \\
\text { Análise dos resultados do uso de Design Centrado no Usuário e do } \\
\text { Design Participativo no design de Sistemas Colaborativos e Assistivos } \\
\text { Registro dos resultados obtidos sobre oportunidades e limites enfrentados } \\
\text { pela utilização de dispositivo móvel de comunicação por pessoas com DI }\end{array}$ \\
\hline $\begin{array}{c}\text { Comunicação } \\
\text { da } \\
\text { Pesquisa }\end{array}$ & $\begin{array}{l}\text { Visa a comunidade científica interessada no desenvolvimento de } \\
\text { Sistemas Colaborativos Assistivos e na Comunicação Aumentativa } \\
\text { e Alternativa }\end{array}$ \\
\hline
\end{tabular}


Em alguns casos, existem condições de realização destes deslocamentos sem a presença de um cuidador, porém os cuidadores não se sentem seguros em deixar que o DI se desloque sozinho. A insegurança é justificada pela possibilidade da ocorrência de problemas no percurso, os quais a pessoa com DI pode ter dificuldades para resolver sozinha. Por exemplo, pegar um ônibus errado, ter uma crise durante o trajeto, sentir medo ou se sentir perdido.

Para [Glat 2004], o cuidado de pessoas com DI impõe adaptações cotidianas. Tais adaptações podem levar muitas famílias ao isolamento social e consequentemente levar a família a reforçar mecanismos de superproteção. [Glat 2004] ainda reforça que, nesses casos, a condição do indivíduo com deficiência pode ser hiperdimensionada em detrimento as suas capacidades e o cuidado pode se tornar exagerado, prejudicando sua independência e autonomia. [Glat 2004] realizou estudos com familiares de pessoas adultas com DI que demonstram a superproteção, evidenciando a necessidade de construção de um espaço ressocializador. A superproteção é evidenciada também por [Rosa and Denari 2012], que encorajam as famílias a oferecer a pessoa com DI maior independência e integração a sociedade.

Neste contexto surgiu a oportunidade de apoiar a autonomia de pessoas com DI por meio do desenvolvimento de um sistema colaborativo de cuidado e localização. Assim, foi idealizado um sistema onde a pessoa com DI e seus cuidadores possam colaborar e dessa forma proporcionar confiança aos cuidadores e um aumento da autonomia de pessoas com DI em seus deslocamentos diários.

$\mathrm{Na}$ busca de possibilitar a colaboração dos cuidadores e dos monitorados (com DI), a presente pesquisa teve foco na definição de artefatos de software de comunicação entendíveis para as pessoas com DI. Basicamente, a comunicação é o processo de transferência de informação e implica na transmissão e recepção de mensagens (pensamentos, ideias, desejos e sentimentos) de uma pessoa para a outra [Tomasello and Tomasello 2003]. As capacidades comunicativas são importantes no desenvolvimento e manutenção das relações sociais e pessoas com DI, que frequentemente têm dificuldades de comunicação.

Portanto, no contexto da pesquisa apresentada nesse artigo e na busca do desenvolvimento de uma Comunicação Aumentativa e Alternativa (CAA), o problema de comunicação relatado indicou a oportunidade de desenvolvimento de artefatos de software que possibilitem que pessoas com DI possam colaborar e se comunicar utilizando dispositivos como smartphones.

\section{Artefatos}

Durante o desenvolvimento da pesquisa foi desenvolvido um sistema de monitoramento. Ele possui um "Módulo de Colaboração" utilizado para a iteração da pessoa com DI com o sistema e portanto, este módulo é o foco da pesquisa. O módulo de colaboração possibilitará a troca de mensagens de texto por chat entre monitorado com DI e os monitores (cuidadores). O foco da pesquisa é mantido nos artefatos que permitem a comunicação. Portanto, são as telas e os componentes de tela do módulo de colaboração, que possibilitarão a comunicação entre os usuários monitores e monitorados.

Os sistemas colaborativos, em geral, são compostos de ferramentas genéricas, tais como: correio-eletrônico, lista de discussão, fórum, chat, entre outros 
[Ugulino et al. 2008]. A Figura 1 traz o exemplo de uma tela de chat para ilustrar a colaboração de Maria e João que representam monitores que se comunicam por mensagens de texto com José que representa o monitorado com DI que se comunica por símbolos. O sistema colabora informando os eventos do monitoramento (horário de saída, chegada, etc).

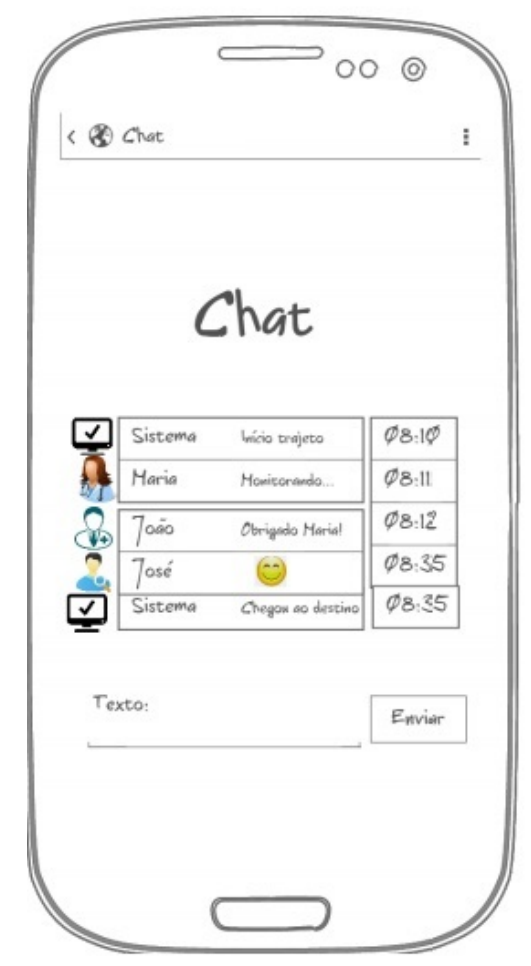

Figura 1. Protótipo de tela de CHAT do módulo de colaboração

\section{Processo de Busca de Solução}

O desenvolvimento da presente pesquisa foi dividida em ciclos de design e para melhor representação, cada ciclo foi dividido em três ciclos de conhecimento. O primeiro ciclo de conhecimento é o ciclo intitulado "conhecendo o usuário" e está detalhado na subseção 6.1 .

\subsection{Ciclo 1 do Design: Conhecendo o Usuário}

A Figura 2 demonstra o ciclo de geração de conhecimento, conforme sugere o DSR, para o primeiro ciclo da busca da solução. A entidade "ciência do comportamento", demonstra o objetivo na busca do conhecimento dos usuários, suas limitações e capacidades. É onde a pesquisa aborda as características dos usuário e como essas características influenciam a utilização do sistema na comunicação.

É utilizada a abordagem de design focado no usuário (UCD, do inglês User Centered Design) para fortalecer a estratégia de foco nas necessidades dos usuários. A entidade "ciência de design" demonstra a prototipação das telas de comunicação do sistema. A prototipação, além de demonstrar a funcionalidade de comunicação em desenvolvimento, proporciona subsídios para avaliação. Com a prototipação é possível avaliar se os artefatos de comunicação em desenvolvimento irão atender as necessidades dos usuários. 
Finalizando o ciclo, a avaliação de especialistas com base no protótipo identifica sucessos e insucessos dos artefatos de comunicação.

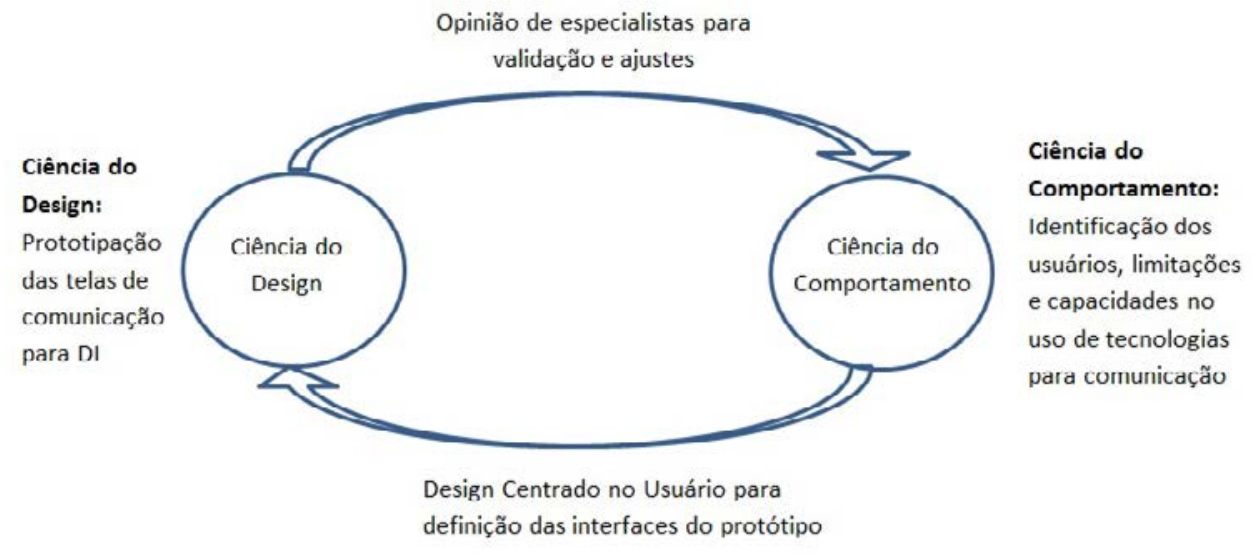

Figura 2. DSR Ciclo 1, adaptado de [Hevner 2007]

O primeiro passo para entendimento dos usuários foi entender a participação de cada usuário na utilização do sistema. Foi realizada um entrevista aberta para a identificação inicial dos usuários e suas relações com o sistema. Para [Minayo 1993], a entrevista aberta é utilizada quando o pesquisador deseja obter a maior quantidade de informações sobre determinado tema, segundo a visão do entrevistado, e também para obter um maior detalhamento do assunto em questão. Ela é utilizada geralmente na descrição de casos individuais, na compreensão de especificidades culturais para determinados grupos e para comparabilidade de diversos casos.

As entrevistas foram realizadas com profissionais ligados a uma instituição que presta apoio terapêutico a pessoas com DI. Foi realizada uma entrevista com um terapeuta ocupacional e outra com um psicólogo, ambos com contato profissional com os usuários foco do sistema. Diversas foram as contribuições providas da execução das entrevistas, podendo-se destacar a identificação da classificação das pessoas com DI de acordo com sua autonomia. Para identificar o grau de DI de seus pacientes, a instituição envolvida na pesquisa, desenvolveu internamente, uma tabela que os classifica de acordo com a capacidade de autonomia e socialização e relaciona essas características a uma determinada cor, conforme apresentado na Tabela 2.

Após as entrevistas um importante requisito do sistema foi definido: Optou-se por definir que os usuários foco da pesquisa são os usuários que se encaixam nas características da classificação azul. Essa definição foi realizada para ir em encontro a um dos objetivo do sistema, que é o aumento da autonomia nos deslocamentos das pessoas com DI, pois nem todos os deficiêntes intelectuais podem se deslocar sozinhos, como demostra a Tabela 2.

\subsection{Ciclo 2 do Design: Definindo os requisitos de usabilidade}

A Figura 3 mostra o ciclo de geração de conhecimento para o segundo ciclo do design.

Neste ciclo, a "ciência do comportamento"é definida pela utilização dos artefatos de comunicação pelas pessoas com DI. Em busca de maior assertividade no de- 
Tabela 2. Tabela de cores para representação da autonomia das pessoas com DI

\begin{tabular}{|l|l|}
\hline Cores & Caracteristicas \\
\hline Vermelho & $\begin{array}{l}\text { Maior grau de dependência } \\
\text { Pouca ou nenhuma autonomia nas tarefas cotidianas } \\
\text { Dificuldades severas de locomoção e comunicação } \\
\text { Baixa ou nenhuma socialização }\end{array}$ \\
\hline Amarelo & $\begin{array}{l}\text { Maior autonomia em relação ao "Vermelho", porém ainda com pouca autonomia } \\
\text { Pouca autonomia nas tarefas cotidianas } \\
\text { Dificuldades de locomoção e comunicação } \\
\text { Baixa socialização }\end{array}$ \\
\hline Verde & $\begin{array}{l}\text { Maior Autonomia em relação ao "Amarelo" } \\
\text { Deficiência intelectual moderada } \\
\text { Habilidades de locomoção e comunicação, mesmo apresentando dificuldades } \\
\text { Possibilidade de socialização }\end{array}$ \\
\hline Azul & $\begin{array}{l}\text { Deficiência intelectual leve } \\
\text { Realiza pequenas decisões } \\
\text { Sociável } \\
\text { Podem ser ou não alfabetizados } \\
\text { Habilidades de comunicação e locomoção, mesmo apresentando dificuldades }\end{array}$ \\
\hline
\end{tabular}

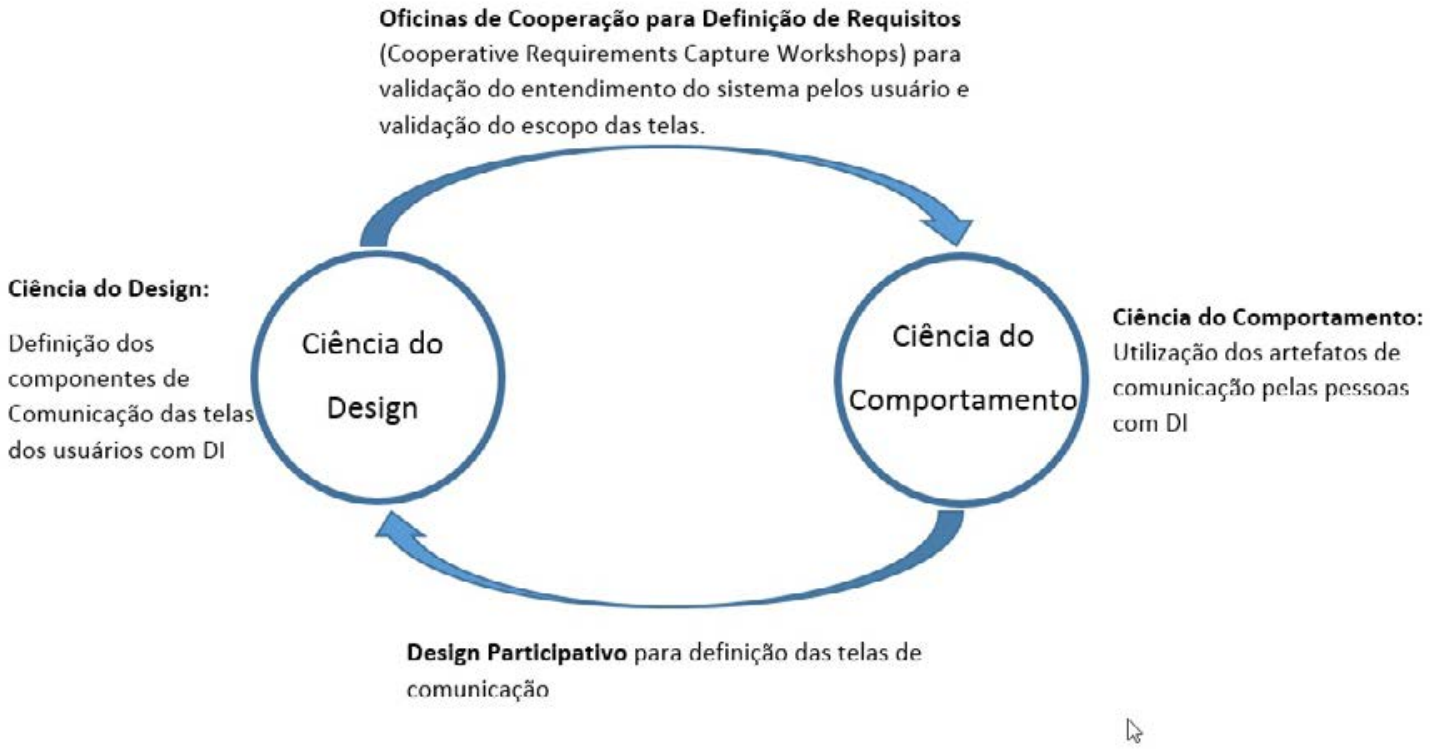

Figura 3. DSR Ciclo 2, adaptado de [Hevner 2007]

senvolvimento dos artefatos de comunicação, a presente pesquisa utilizou da metodologia de desenvolvimento de sistemas denominada Design Participativo (DP). Para [Muller et al. 1997], o DP envolve um conjunto de teorias, práticas e estudos relacionados com utilizadores (profissionais e trabalhadores) no desenvolvimento de software, hardware ou qualquer atividade relacionada com o computador. A realização de workshops foi a prática escolhida do DP para envolvimento dos usuários na definição dos artefatos de comunicação. Já a entidade "ciência de design"demonstra o refinamento das telas 
de prototipação do sistema. Os workshops possibilitaram a avaliação da usabilidade dos componentes de tela dos protótipos.

Para ser mais específico, foi utilizada a prática de Oficinas de Cooperação para Definição de Requisitos, do inglês Cooperative Requirements Capture Workshops. Baseada em uma análise de quem são os as partes interessadas em um sistema de computador e quais os problemas de projeto, essa técnica possibilita aos profissionais de software realizarem sete passos para determinar os requisitos de design. Três etapas envolvem o trabalho direto com os usuários e as demais fornecem o contexto dentro do ciclo de vida do desenvolvimento. Os sete passos são respectivamente: análise de partes interessadas, identificação do problema, formular a equipe de entre as quatro classes de partes interessadas (descritas no modelo de participação da técnica), explorar o ambiente do usuário por meio de uma oficina (User Workshop), validar o entendimento de ambiente de usuário, identificar o escopo da solução e validar o escopo.

A Engenharia Semiótica foi proposta por [De Souza 2005] para o design de linguagens de interface e tem como base teórica a semiótica, disciplina que estuda signos e linguagens de produção de significado e sentido [da Rocha and Baranauskas 2003]. Para [Barbosa and da Silva 2010] com o intuito de tornar as interfaces amigáveis e naturais, e assim, mais fáceis de serem usadas e menos hostis, são utilizados elementos gráficos para representar comandos, dados ou funções do sistema. [Barbosa and da Silva 2010] ainda definem que em uma interface de tela, um signo é uma mensagem codificada pelo designer para se comunicar com usuário.

O objetivo do workshop é trabalhar com os usuários com DI, buscando a identificação do melhor entendimento das telas de comunicação do sistemas voltadas para estes usuário. Ou seja, é utilizar o workshop como ferramenta do DP, para definir os artefatos gráficos das telas de comunicação utilizadas pelos usuários com deficiência intelectual, visando a usabilidade e possibilitando assim comunicação.

\subsection{Ciclo 3 do Design: Avaliando a Usabilidade}

O terceiro ciclo do design busca avaliar se o emprego de técnicas do Design Centrado no Usuário e do Design Participativo, durante as fases de levantamento de requisitos e desenvolvimento da solução, resultaram em uma boa usabilidade do sistema por parte das pessoas com deficiência intelectual.

Um ambiente controlado será preparado para a avaliação da usabilidade. A usabilidade será medida por duas técnicas: A observação do uso e a utilização de componentes. O primeiro se dá pela observação da utilização do sistema pelo usuário e cabe ao observador avaliar o entendimento e capacidade de utilização do usuário. Já a avaliação de utilização dos componentes será a análise dos registros da utilização $(\log s)$ e das mensagens do chat.

A Figura 4 mostra o ciclo de geração de conhecimento para o terceiro e último ciclo do design.

\section{Rigor da Pesquisa}

De acordo com [Thomas and Hatchuel 2009], para que uma pesquisa seja confiável, ela deve se preocupar não somente com a relevância, mas também com o rigor, que deve estar presente desde a sua condução até a apresentação de seus resultados. 


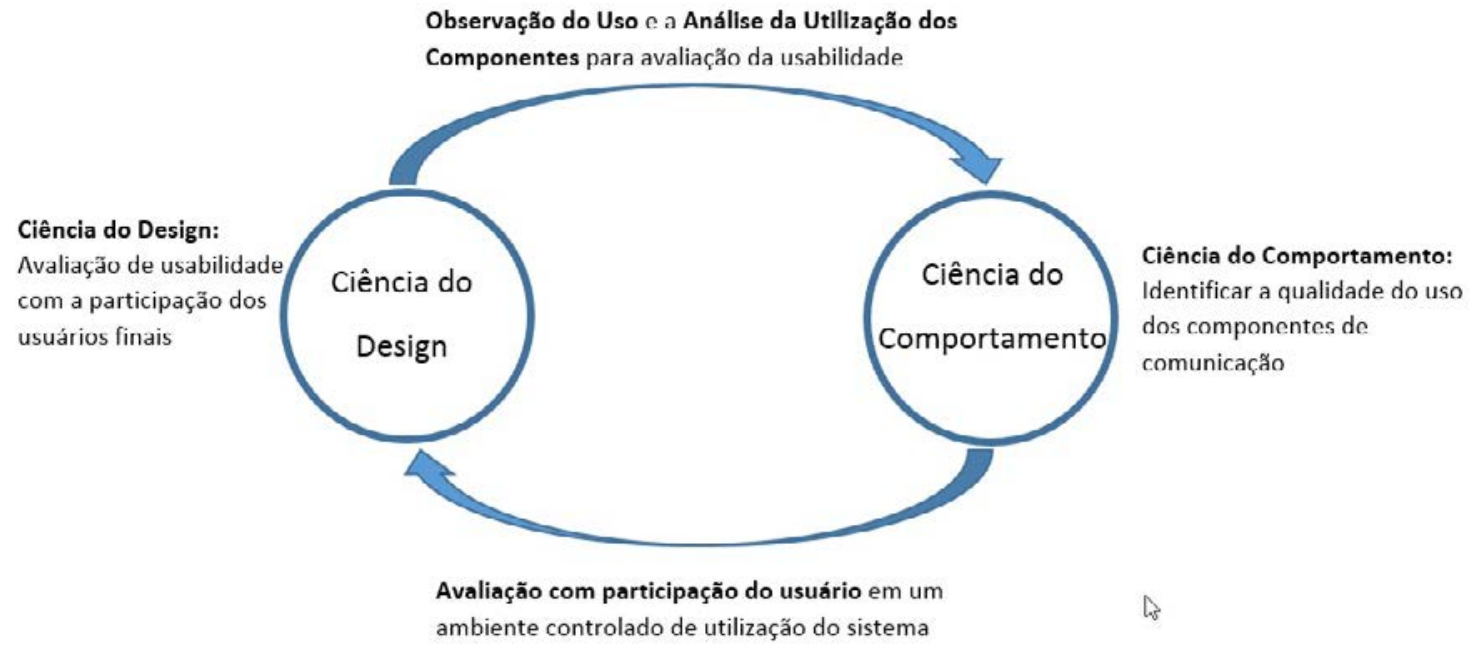

Figura 4. DSR Ciclo 3, adaptado de [Hevner 2007]

O rigor da presente pesquisa está registrada nas Figuras 2, 3 e 4, na opinião de especialistas, oficinas de cooperação e na observação de uso e análise dos componentes, respectivamente.

O primeiro ciclo desta pesquisa resultou na construção de protótipos das telas de comunicação do sistema com base nos conceitos da abordagem UCD. A validação do artefato gerado nesse ciclo foi um método de avaliação analítico chamado Opinião de Especialistas. Para [Prates and Barbosa 2003], métodos de avaliação analíticos são aqueles nos quais avaliadores inspecionam ou examinam aspectos de uma interface de usuário relacionados com a usabilidade.

\section{Conclusão e Trabalhos Futuros}

A presente pesquisa contempla a realização do design da comunicação em um sistema colaborativo de monitoramento de pessoas com Deficiência Intelectual (DI), produzindo artefatos que possibilitem a comunicação de pessoas com DI com seus cuidadores. Pretende-se assim, proporcionar confiança aos cuidadores e um aumento da autonomia de pessoas com DI em seus deslocamentos diários. A pesquisa foi guiada pela metodologia de pesquisa Design Science Research (DSR) e foi dividida tem três ciclos de descoberta de conhecimento.

No primeiro ciclo foi gerado um protótipo de baixa fidelidade como artefato da "ciência do Design". A "ciência do comportamento", do primeiro ciclo, é definida pela necessidade de conhecimento dos usuários, que no contexto deste estudo, são usuários com capacidades cognitivas reduzidas. O primeiro ciclo se caracterizou pela utilização da abordagem do Design Centrado no Usuário.

O segundo ciclo tem o objetivo de validação e ajustes no protótipo obtido no ciclo 1. Os artefatos do ciclo 2 são os componentes de tela de comunicação, utilizadas pelos usuários com deficiência intelectual e definidos com o apoio destes usuários e baseados 
nas abordagens do Design Participativo. Nessa etapa, foram utilizadas como ferramenta as Oficinas de Cooperação para Definição de Requisitos.

O terceiro ciclo da descoberta de conhecimento será executado. A definição do terceiro ciclo está descrita na seção 6.3 do presente artigo, porém os resultados ainda não foram coletados e analisados. O terceiro ciclo usa o resultado da avaliação de usabilidade como o artefato da ciência do design e utiliza um ambiente controlado de utilização para avaliação por observação de uso e utilização de componentes do sistemas $(\log s)$.

Podemos concluir que o DSR tem potencial para produção de conhecimento no processo de design de sistema colaborativo e assistivo. Destaca-se também a importância das abordagens de Design Centrado no Usuário e do Design participativo como ferramenta para o design da comunicação aumentativa e alternativa no contexto do sistema proposto.

Como trabalhos futuros, a avaliação de usabilidade, em um ambiente controlado de uso, possibilitará a validação das ferramentas utilizadas para a construção do sistema, assim como trará uma nova contribuição científica com a análise dos resultados da avaliação.

\section{Referências}

Aken, J. E. v. (2004). Management research based on the paradigm of the design sciences: the quest for field-tested and grounded technological rules. Journal of management studies, 41(2):219-246.

Almeida, M. S. R. (2005). Caminhos para a inclusão humana. Porto: Edições Asa.

Barbosa, S. D. J. and da Silva, B. S. (2010). Interação humano-computador. Elsevier.

Bax, M. P. (2015). Design science: filosofia da pesquisa em ciência da informação e tecnologia. Ciência da Informação, 42(2).

Chang, Y.-J., Liu, H.-H., and Wang, T.-Y. (2009). Mobile social networks as quality of life technology for people with severe mental illness. Wireless Communications, IEEE, 16(3):34-40.

da Rocha, H. V. and Baranauskas, M. C. C. (2003). Design e avaliação de interfaces humano-computador. Unicamp.

de Araújo, L. P., Berkenbrock, C. D. M., and Mattos, M. M. (2014). Usando pesquisaação no desenvolvimento de um sistema colaborativo para tratamento multidisciplinar na rede do sus.

De Souza, C. S. (2005). The semiotic engineering of human-computer interaction. MIT press.

Druin, A. (2009). Mobile technology for children: Designing for interaction and learning. Morgan Kaufmann.

Fink, P. J. (1992). Stigma and mental illness. American Psychiatric Pub.

Fuks, H. (2011). Sistemas colaborativos. Elsevier Brasil.

Glat, R. (2004). Uma família presente e participativa: o papel da família no desenvolvimento e inclusão social da pessoa com necessidades especiais. In Anais do $9^{\circ}$ Congresso Estadual das APAEs de Minas Gerais, pages 1-7. 
Grudin, J. (1994). Computer-supported cooperative work: History and focus. Computer, (5):19-26.

Hevner, A. R. (2007). A three cycle view of design science research. Scandinavian journal of information systems, 19(2):4.

Lacerda, D. P., Dresch, A., Proença, A., and ANTUNES JUNIOR, J. (2013). Design science research: método de pesquisa para a engenharia de produção. Gestão \& Produção, 20(4):741-761.

Minayo, M. C. d. S. (1993). O desafio do conhecimento científico: pesquisa qualitativa em saúde. São Paulo/Rio de Janeiro: Hucitec-Abrasco, pages 01-10.

Muller, M. J., Haslwanter, J. H., and Dayton, T. (1997). Participatory practices in the software lifecycle. Handbook of human-computer interaction, 2:255-297.

Petrie, H., Hamilton, F., King, N., and Pavan, P. (2006). Remote usability evaluations with disabled people. In Proceedings of the SIGCHI Conference on Human Factors in Computing Systems, CHI '06, pages 1133-1141, New York, NY, USA. ACM.

Prates, R. O. and Barbosa, S. D. J. (2003). Avaliação de interfaces de usuário-conceitos e métodos. In Jornada de Atualização em Informática do Congresso da Sociedade Brasileira de Computação, Capítulo, volume 6.

Pries-Heje, J., Baskerville, R., and Venable, J. (2008). Strategies for design science research evaluation. ECIS 2008 proceedings, pages 1-12.

Radabaugh, M. (1993). Selecting and obtaining assistive technology-ibm national support center for persons with disabilities. Interagency Project for assistive technologyIPAT. North Dakota: IPAT.

Rocha, E. B., Pimentel, M., Diniz, M. C., and Santoro, F. M. (2015). Design science research para o desenvolvimento de um modelo da participação em bate-papo. iSysRevista Brasileira de Sistemas de Informação, 8(1):18-41.

Rosa, F. D. and Denari, F. E. (2012). Trabalho, educação e família: perspectivas para a pessoa com deficiência intelectual. Revista Educação Especial, 26(45):73-90.

Satcher, D. (2000). Mental health: A report of the surgeon general-executive summary. Professional Psychology: Research and Practice, 31(1):5.

SNPD (2012). Cartilha do censo 2010 - pessoas com deficiências.

Thomas, H. and Hatchuel, A. (2009). A foundationalist perspective for management research: A european trend and experience. Management Decision, 47(9):1458-1475.

Tomasello, M. and Tomasello, M. (2003). Comunicação linguística e representação simbólica. M. Tomasello, Origens culturais da aquisição do conhecimento humano, pages $131-186$.

Ugulino, W., Nunes, R. R., Oliveira, C. L., Pimentel, M., and Santoro, F. M. (2008). Dos processos de colaboração para as ferramentas: A abordagem de desenvolvimento do projeto communicatec. In Companion Proceedings of the XIV Brazilian Symposium on Multimedia and the Web, WebMedia '08, pages 233-240, New York, NY, USA. ACM.

Wahl, O. F. (1999). Mental health consumers' experience of stigma. Schizophrenia bulletin, 25(3):467. 